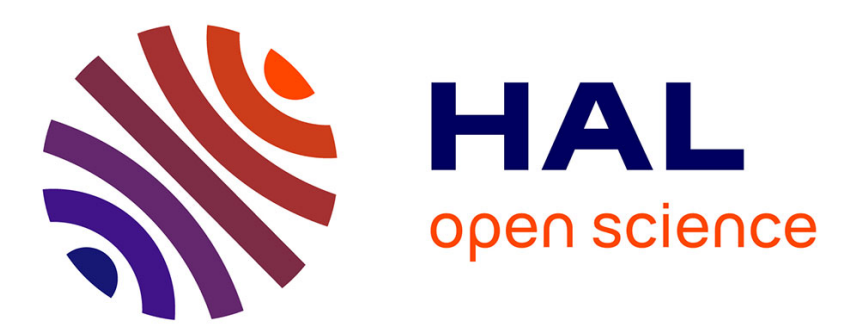

\title{
Syncrétisme des formes verbales et des clitiques sujets dans plusieurs variétés romanes vernaculaires et en français standard

\author{
Jean Sibille
}

\section{- To cite this version:}

Jean Sibille. Syncrétisme des formes verbales et des clitiques sujets dans plusieurs variétés romanes vernaculaires et en français standard. Internationalen Arbeitstagung zum romanisch-deutschen und innerromanischen Sprachvergleich, Université d'Innsbruck (Autriche), Sep 2012, Innsbruck, Autriche. pp.341-354. hal-01297204

\section{HAL Id: hal-01297204 \\ https://hal.science/hal-01297204}

Submitted on 3 Apr 2016

HAL is a multi-disciplinary open access archive for the deposit and dissemination of scientific research documents, whether they are published or not. The documents may come from teaching and research institutions in France or abroad, or from public or private research centers.
L'archive ouverte pluridisciplinaire HAL, est destinée au dépôt et à la diffusion de documents scientifiques de niveau recherche, publiés ou non, émanant des établissements d'enseignement et de recherche français ou étrangers, des laboratoires publics ou privés. 


\section{SYNCRÉTISME DES FORMES VERBALES ET DES CLITIQUES SUJETS DANS PLUSIEURS VARIÉTÉS ROMANES VERNACULAIRES ET EN FRANÇAIS STANDARD}

In diesem Aufsatz wird eine kontrastive Analyse des Klitika-Endungssystems im Standardfranzösischen und in mehreren romanischen Regionalvarietäten, die vollständige (Piemontesisch, okzitanische Mundarten von Chiomonte und Ramats (Italien)) oder partielle Subjektklitikaparadigmen (ligurische Mundarten von Bonifacio und Haute Roya) besitzen, vorgelegt. Aus der Analyse wird erkenntlich, dass in den Systemen, die Synkretismus sowohl in den Verbalendungen als auch im Subjektklitikaparadigma aufweisen, die Verbindung der Marker (Klitikum + Endung) die Markierung hervorruft und dass zwischen strikten Pro-DropGrammatiken und strikten Nicht-Pro-Drop-Grammatiken ein Kontinuum existiert, in dem mehrere Variablen ins Spiel kommen. Daher bietet es sich an, in dieser Varietätenart das Klitika-Endungssystem als ein Ganzes, das ein- und dasselbe Markierungssystem darstellt, zu analysieren.

Cet article propose une analyse contrastive du système clitiques-désinences en français standard et dans plusieurs variétés romanes vernaculaires possédant des paradigmes complets de clitiques sujet: piémontais, occitan de Chiomonte et des Ramats (Italie); ou des paradigmes partiels : parlers liguriens de Bonifacio et de la Haute Roya. Il ressort de l'analyse que, dans les systèmes présentant du syncrétisme à la fois dans les désinences verbales et dans le paradigme des clitiques sujets, c'est l'association des marques (clitique + désinence) qui produit le marquage, et que, entre grammaires strictement pro drop et grammaires strictement non pro drop, il existe un continuum dans lequel plusieurs variables entrent en jeu. C'est pourquoi il convient, dans ce type de variété, d'analyser le système clitiques-désinences comme un tout constituant un seul et même système de marquage.

\section{Introduction}

Parmi les langues romanes standard, seul le français conjugue les verbes avec un clitique sujet: l'italien, l'espagnol, le portugais et le roumain ont une flexion verbale dite pro drop. Si l'on élargit l'analyse aux variétés vernaculaires, il apparait que de nombreuses variétés romanes ont développé des systèmes complets ou partiels - de clitiques sujets : parlers francoprovençaux, galloitaliques (piémontais, lombard, ligurien, émilien, romagnol), romanches, ladins frioulans. En ce qui concerne l'occitan, la plupart des parlers ont une flexion pro drop, toutefois certaines variétés situées sur les marges du domaine, au nord et à 
l'est, dans des zones de contact avec des systèmes non pro drop, ont développé des paradigmes de clitique sujets.

Plusieurs questions théoriques concernant les clitiques sujets sont en débat au sein de la communauté des linguistes :

- Les clitiques ont-ils un statut lexical ou un statut morphologique ? En d'autres termes : sont-ils des arguments pronominalisés ou des marqueurs d'accord ? 1

- D'un point de vue diachronique, la position classique consiste à considérer que les clitiques sujets sont venu compenser l'usure phonétique des désinences (Meyer-Lübke 1895) mais pour Adams (1988), c'est le développement des paradigmes de clitiques sujets qui permet l'usure des désinences.

- Existe-t-il un paramètre du sujet nul dans la grammaire universelle ? La théorie générativiste postule l'existence d'une gamme a priori restreinte de possibilités structurelles permettant au petit enfant de déchiffrer la grammaire sous-jacente dans le discours de ses parents en limitant le nombre des hypothèses qu'il doit explorer. Dans cette perspective, il existerait une distinction binaire radicale entre grammaires à sujet nul et grammaires à sujet obligatoire.

Nous proposons ici une analyse fonctionnelle du marquage de la personne du verbe au niveau des structures de surface, en synchronie et d'un point de vue morphosyntaxique, c'est-à-dire dans le cadre d'une approche contextualisée de la morphologie. Cette démarche est basée sur le questionnement suivant: "Quelle marque ${ }^{2}$ apporte quelle information? Comment est construit le marquage de la personne? ».

Dans le cadre de cette démarche, la réponse aux deux premières questions ci-dessus (statut lexical ou morphologique des clitiques et compensation en diachronie, par les clitiques, de l'usure de la désinence) n'a que peu d'importance. En revanche, nous reviendrons dans la conclusion sur la question du paramètre du sujet nul.

Dans certains systèmes on constate que d'un point de vue informatif, en synchronie, la présence de clitiques sujets compense le syncrétisme de certaines

\footnotetext{
${ }^{1} \mathrm{La}$ réponse à cette question peut être variable suivant les langues. La plupart des auteurs semblent s'accorder pour considérer qu'en anglais, les pronoms sujets ont un statut lexical (cf. notamment Cabredo-Hofherr 2004). Pour ce qui est du français, le statut lexical des clitiques est défendu notamment par Kayne (1983) et De Cat (2005), le statut morphologique par ZribiHertz (1994) et Miller / Monachesi (2003). Pour les variétés gallo-italiques, Rizzi (1986) considère que, dans la mesure où le clitique sujet double obligatoirement le sujet lexical, il a un statut morphologique.

2 Nous appelons marque tout élément apportant une information de type grammatical : morphème isolable, trait suprasegmental, modification de la base (flexion interne), voire élément lexical.
} 
désinences. Il s'agit là d'un fait objectif. Pour autant, cela ne signifie pas que tout système dans lequel apparaissent des désinences syncrétiques développe automatiquement un système de clitiques sujets.

En effet, certains systèmes admettent une dose modérée de syncrétisme : par exemple, en espagnol decía signifie 'je disais' ou 'il disait' ; en italien canti, est la deuxième personne du présent de l'indicatif de cantare 'chanter' mais aussi la première, deuxième ou troisième personne du présent du subjonctif ; dans la plupart des parlers languedociens, [kant'aßes] signifie 'tu chantais' ou 'vous chantiez'. Dans de tels cas la personne est identifiée par le contexte ou peut toujours être précisée par un pronom sujet.

D'autres systèmes ont pu réagir au syncrétisme en diversifiant les désinences. C'est ainsi qu'en occitan médiéval parlava signifiait 'je parlais' ou 'il parlait', alors qu'en occitan moderne on a : parlavi [parl'a $\mathrm{ai}]^{3}$ 'je parlais' et parlava [parl'aßo] 'il parlait'.

Il faut également souligner que les systèmes dans lesquels certaines désinences sont devenues homophones ne sont pas les seuls à développer des paradigmes de clitiques sujets. Certains systèmes conservant des désinences verbales bien distinctes on développé de tels paradigmes, comme par exemple le parler francoprovençal de Mezzenile (Italie, province de Turin) :

\begin{tabular}{|c|c|}
\hline $\begin{array}{l}\varnothing \text { [dy'ərmu] 'je dors' } \\
\text { [et dyər] 'tu dors' } \\
\mathbf{u} / \mathbf{e} \text { [dy' ort] 'il/elle dort', } \\
\varnothing \text { [dyrm' } \varepsilon \text { ] ' 'nous dormons', } \\
\text { [u dyrm'i:] 'vous dormez', } \\
\text { [u/e dy' ormunt]'ils/elles dorment'/ }\end{array}$ & 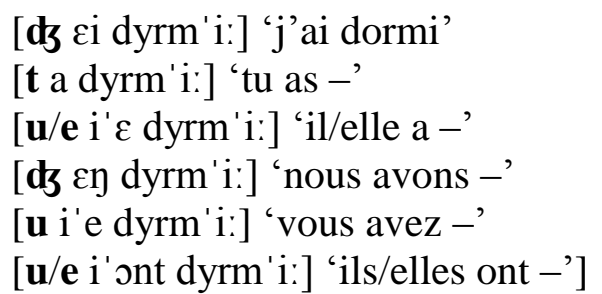 \\
\hline
\end{tabular}

(Source : Floricic / Molinu 2008)

Nous examinerons dans un premier temps des systèmes à paradigme partiel de clitiques sujets : le parler ligurien de Bonifacio à un seul clitique et les parlers liguriens alpins de la haute vallée de la Roya qui possèdent des systèmes à deux ou trois clitiques. Nous analyserons ensuite trois systèmes à paradigme complet : le français standard, le piémontais turinois, et le parler occitan de Chiomonte et des Ramats (Italie, province de Turin).

La commune de Chiomonte (anciennement Chaumont), dans la Haute Vallée de Suse, est la dernière commune occitanophone à la fois au nord et à l'est ; à Gravère à trois $\mathrm{km}$ on parle francoprovençal et la ville de Suse, à $7 \mathrm{~km}$, est une enclave piémontaise en zone francoprovençale. La commune est composée du bourg de Chiomonte et des hameaux des Ramats, plus isolés dans la montagne, à 4 ou $5 \mathrm{~km}$ du bourg. Il existe quelques différences entre le parler de Chiomonte et celui des Ramats - notamment, comme on le verra, dans le

\footnotetext{
${ }^{3} \mathrm{Ou}$ parlave. Certains parlers limousins ou ariégeois, toutefois, conservent parlava 'je parlais'.
} 
paradigme des clitiques sujets - mais qui ne sont pas de nature à entraver l'intercompréhension. Les données concernant Chiomonte et Les Ramats, utilisées dans le présent article, sont des données primaires provenant d'enquêtes effectuées en 2011 et 2012 et d'enregistrements d'interviews effectués en 1984.

\section{Systèmes à paradigme partiel}

\subsection{Système à un seul clitique : Bonifacio}

Le parler ligurien de Bonifacio, en Corse, représente un exemple de système à un seul clitique :

Tableau 1 : Bonifacio, flexion verbale ${ }^{4}$ (source : Comiti 1994)

\begin{tabular}{|c|c|c|c|c|c|}
\hline \multicolumn{2}{|c|}{ cantà 'chanter' ( 1 ère conjugaison) } & \multicolumn{3}{l|}{ vendi 'vendre' $\left(3^{\text {ème }}\right.$ conjugaison) } \\
\hline Prés. ind. & Futur & Subjonctif & Prés. ind. & Futur & Subjonctif \\
\hline cantu & cantirò & cantessi & vendu & vindirò & vindessi \\
\hline ti canti & ti cantiré & ti cantessi & ti vendi & ti vindiré & ti vindessi \\
\hline canta & cantirà & cantessi & vendi & vindirà & vindessi \\
\hline cantemu & cantiremu & cantéssimu & vindimu & vindiremu & vindéssimu \\
\hline cantè & cantiré & cantessi & vindii & vindiré & vindessi \\
\hline càntinu & cantiranu & cantessinu & véndinu & vindiranu & vindessinu \\
\hline
\end{tabular}

La présence du clitique ti lève l'ambigüité entre les pers. 2 et 3 du présent de l'indicatif des $2^{\text {ème }}$ et $3^{\text {ème }}$ conjugaisons, les pers. 2 et 4 du futur et de l'imparfait. Elle permet également de distinguer la pers. 2 du subjonctif et du conditionnel des pers. 1,3 et 5 qui, elles, restent homophones.

Le clitique $t i$ est obligatoire. Il n'est pas formellement distinct du pronom tonique, néanmoins c'est un véritable clitique qui peut être cumulé avec le pronom tonique thématisé ou détaché à droite : $t i$, $t i$ canti 'toi, tu chantes'; $t i$ canti, $t i$ 'tu chantes, toi'. Le clitique complément de pers. 2 a également la forme $t i$; à la forme pronominale on aura donc : ti ti lavi senza savun 'tu te laves sans savon', $t i$, ti ti lavi senza savoun 'toi, tu te laves sans savon'; ou, avec un datif étendu : $t i$ ti mangi u frumangiu senza pan litt. 'tu te manges le fromage sans pain', $t i$, ti ti mangi u frumangiu senza pan litt. 'toi, tu te manges le fromage sans pain'. Dans les relatives, $t i$ reste obligatoire : $t i$ sé $t i$ che ti canti cun mi 'c'est toi qui chantes avec moi' (litt. 'tu es toi que tu chantes avec moi')

\footnotetext{
${ }^{4}$ En ce qui concerne le syncrétisme, l'imparfait a la même structure paradigmatique que le futur, et le conditionnel a la même structure que le subjonctif.

${ }^{5}$ Données communiquées par Jean-Marie Comiti, Università di Corsica - Pasquale Paoli.
} 


\subsection{Systèmes à deux ou trois clitiques (Haute Roya)}

Les parlers liguriens alpins de la haute vallée de la Roya ${ }^{6}$ présentent un système à deux ou trois clitiques sujets avec distinction du genre aux personnes 3 et 6 , comme le montrent les tableaux ci-dessous (source Dalbera 1991 et 1994, ainsi que Botton et al. 2000 pour Saorge) :

Tableau 2 : Hte Roya, présent de l'indicatif ${ }^{7}$

\begin{tabular}{|c|c|c|c|}
\hline Breil & La Brigue & Tende & Saorge \\
\hline k'anti & $\mathrm{k}^{\prime}$ aant & k'antu & (e) k'antə \\
\hline $\mathrm{k}^{\prime}$ ante & k'antu & k'anta & k'anta \\
\hline aR/a k' anta & or/a k'anta & $\mathrm{a} . \mathrm{s} / \mathrm{a} \mathbf{k}^{\prime} \mathbf{a n t a}$ & eə/a k'anta \\
\hline kant'ama & kant' & kant'amu & (e) kal \\
\hline kant'e & kant'aj & kant'aj & (e) kant'aj \\
\hline $\mathrm{k}^{\prime}$ antu & 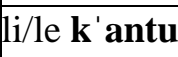 & 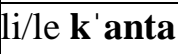 & (e) $\mathrm{k}^{\prime}$ \\
\hline
\end{tabular}

Tableau 3 : Hte Roya, présent du subjonctif ${ }^{8}$

\begin{tabular}{|c|c|c|c|}
\hline Breil & La Brigue & Tende & Saorge \\
\hline k'aant & k'aant & k'ante & (e) k'anta \\
\hline k'ante & $\boldsymbol{k}^{\prime}$ antut & k'ante & k'anta \\
\hline ar/a k'aant & ər/a k'aa & a.s/a k'ante & eə/a k'anta \\
\hline kant'am & kant'em & kant'emu & (e) kant'ame \\
\hline kant'e & kant'e & kant'ej & (e) kantaj \\
\hline $\mathrm{k}^{\prime}$ antu & li/le $\boldsymbol{k}$ 'antu & li/le k'ante & (e) kantan \\
\hline
\end{tabular}

Tableau 4 : Hte Roya, futur

\begin{tabular}{|c|c|c|c|}
\hline Breil & La Brigue & Tende & Saorge \\
\hline $\operatorname{kant}^{\ominus} \mathrm{I}^{\prime} \mathrm{aj}$ & kantəx'aj & kante $\iota^{\prime a j}$ & (e) kantəı'aj \\
\hline kant $^{2} \mathrm{I}^{\prime}$ aa & kantə.I'a & ti $\quad$ kante.ı'a & kantə.' a \\
\hline aR/a kant ${ }^{2} I^{\prime} a$ & כR/a kantə.ı'a & aı/a kante.ı'a & eə/a kantə.ı'a \\
\hline $\operatorname{kant}^{2}{ }^{\prime}{ }^{\prime}$ ema & kantəı' ema & kante.ı'emu & (e) kantəı' emə \\
\hline $\operatorname{kant}^{2} \mathrm{I}^{\prime} \mathrm{e}$ & kantə.ı'e & kante..'ei & (e) kantว.' ei \\
\hline $\operatorname{kant}^{\circ} x^{\prime}$ aan & i/le kantəı' a & le kante. & (e) kantəı' an \\
\hline
\end{tabular}

On constate que, à La Brigue et à Tende, les clitiques sujets sont présents exclusivement à des personnes présentant des formes syncrétiques. Il en va de même à Saorge, si l'on ne considère que les clitiques spécifiques à une seule

\footnotetext{
${ }^{6}$ La Vallée de la Roya débouche à Vintimille en Italie. La partie basse de la vallée se trouve en territoire italien, la partie haute en territoire français.

${ }^{7}$ Pour Saorge, Dalbera ne précise pas que la particule [e] est facultative, mais cela est indiqué dans Botton et al. et ressort très clairement des exemples et des textes présentés. Pour le clitique masculin de pers. 3, Dalbera donne [ə] tandis que Botton et al. donnent éheu [eə].

${ }^{8}$ Dans les quatre variétés, l'imparfait, le conditionnel et le subjonctif imparfait présentent, pour ce qui est du syncrétisme, la même structure paradigmatique que le subjonctif présent.
} 
personne, mais dans ce parler les verbes aux personnes autres que 2 et 3 peuvent être précédées d'une particule facultative [e] qui vient en quelque sorte remplir une « case vide», et que l'on hésite à qualifier de clitique sujet ${ }^{9}$.

À Breil, le clitique de pers. 3 permet de différencier les personnes 1 et 3 du présent et de l'imparfait du subjonctif, ainsi que du conditionnel. Dans ce parler la pers. 2 présente, dans tous les paradigmes, une forme spécifique; la présence du clitique [ti] n'est donc pas nécessaire à l'identification de la pers. 2 . Si l'on tient compte de ce que les différents parler de la vallée, linguistiquement proches, évoluent en interaction les uns avec les autres, il n'est pas étonnant que le parler de Breil utilise, comme les autres parlers de la vallée, un clitique sujet à la pers. 2. De plus, ce fait est conforme aux généralisations sur la hiérarchie des personnes, qui ont pu être dégagées dans différents travaux (Vanelli 1998, Poletto 1993 et 1995, Manzini / Savoia 2005 et 2010, Savoia / Manzini 2010, Heap 2000) $)^{10}$ :

La deuxième personne du singulier représente la forme le plus souvent marquée et différenciée dans les paradigmes de clitiques sujet. Celle-ci est suivie de la troisième personne du singulier, la moins souvent différenciée étant la première personne du singulier. On a donc affaire à une hiérarchie qui correspond au schéma $[. .]:. 2^{\text {ème }} \mathrm{sg}$. > $3^{\text {ème }} \mathrm{sg}$. $>1^{\text {ère }} \mathrm{sg}$. / Si en somme un système de clitiques sujet présente un seul clitique sujet différencié, on peut s'attendre à ce que ce soit le clitique de deuxième personne du singulier [...]. Et si l'on relève une différenciation des clitiques sujet à la troisième du singulier, on s'attend a priori à ce qu'il y ait également un clitique différencié à la deuxième du singulier. (Floricic / Molinu 2008, 46-47)

Autant qu'on puisse en juger à travers la documentation disponible, en tout cas pour Saorge ${ }^{11}$, le clitique sujet de pers. 3 n'est pas utilisé en présence d'un sujet lexical ni après un relatif sujet ${ }^{12}$ : Eu poustilloun passava dïi viadji kada di [ø pustij' un pas'ava d'yi vj'adzi k'ada di] 'Le facteur passait deux fois chaque jour'; Ŕi duganéheu an pilla æun sfozaouheu keu poueutava deheu riheuje [..i dygan'eə an pij'a œn sfoza'uə kø puət'ava døə r' iəzə] 'Les douaniers ont pris un contrebandier qui transportait du riz' (Botton et al., 2, 253)

\section{Systèmes à paradigme complet de clitiques sujet}

\footnotetext{
${ }^{9}$ Floricic / Molinu (2008) qualifient ce type d'élément de zéro morphologique.

${ }^{10}$ Heap (2000) nuance ces généralisations en insistant sur le fait qu'il s'agit plus de tendances que de règles absolues.

${ }^{11}$ Botton et al. (2000) comporte quelques textes et de nombreux exemples de phrases, dans lesquels le clitique n'apparaît jamais en présence d'un sujet lexical.

${ }^{12}$ Par commodité de langage, nous utilisons le terme relatif sujet, bien que cette notion puisse être contesté dans des variétés où l'on a affaire à un relateur invariable.
} 


\subsection{Structure des paradigmes}

Les trois systèmes : français, occitan de Chiomonte et des Ramats, piémontais turinois, présentent des faits de syncrétisme aussi bien dans le paradigme des cliques sujets que dans les formes verbales.

En français le syncrétisme des clitiques sujets est très limité puisqu'il ne concerne que les formes préconsonantiques des clitiques de pers. 3 et $6:$ [i(l)] (masc.), [عl] (fém.); dans les autres variétés étudiées on rencontre les formes suivantes (les formes syncrétiques sont figurées en gras) :

Tableau 5 : clitiques sujets formes préconsonantiques

\begin{tabular}{|c|c|c|c|c:c|c|c|c|c|}
\hline & P1 & P2 & P3m & P3f & P3n & P4 & P5 & P6m & P6f \\
\hline Chiomonte & a & at & u & i & la & nu & $\mathbf{u}$ & i & la: \\
\hline Ramats & a & at & u & i & la & a & u & i & la: \\
\hline piémontais & i & it & \multicolumn{3}{|c|}{ a } & & i & i & \multicolumn{2}{|c|}{ a } \\
\hline
\end{tabular}

Tableau 6 : clitiques sujets formes prévocaliques ${ }^{13}$

\begin{tabular}{|c|c|c|c|c:c|c|c|c|c|}
\hline & P1 & P2 & P3m & P3f & P3n & P4 & P5 & P6m & P6f \\
\hline Chiomonte & a & atl t & ul & il & l & $($ nu $) \mathbf{l}$ & ul & il & laz \\
\hline Ramats & $\varnothing$ & t & ul & il & 1 & $\varnothing$ & ul & il & laz \\
\hline
\end{tabular}

Le parler de Chiomonte possède un système complet de clitiques tant en position préconsonantique qu'en position prévocalique, tandis qu'aux Ramats le clitique sujet des pers. 1 et 4 est nul devant voyelle. Le piémontais n'opère pas de distinction de genre aux pers. 3 et 6 , tandis que l'occitan de Chiomonte et des Ramats possède à la pers. 3 non seulement un clitique masculin et un clitique féminin, mais aussi un clitique neutre ${ }^{14}$. En français certains pronoms toniques ont une forme différente de celle du clitique ; ceci est systématique dans les autres variétés étudiées. Dans tous les cas, le clitique peut être cumulé avec le pronom tonique correspondant, thématisé ou détaché à droite, français : 'moi, je', 'toi, tu', 'lui, il', 'elle, elle' etc. ; Ramats : [mi a], [ty t] [je: u] [j'ali i] etc. ; piémontais : [mi i], [ti it], [kjel a], [k' ila a] etc.

Le français tend vers un système où la personne est marquée par le clitique et le temps-mode par la désinence ${ }^{15}$. En revanche dans l'occitan de Chiomonte et des Ramats, ainsi qu'en piémontais, on constate une même

\footnotetext{
${ }^{13}$ En piémontais le paradigme est identique devant consonne et devant voyelle, sauf devant les verbes 'être' et 'avoir' avec lesquels les clitiques prévocaliques ont une forme particulière.

${ }^{14}$ Faute de mieux, nous préférons parler de clitique neutre plutôt que de clitique explétif, car s'il est explétif lorsqu'il est employé avec un verbe impersonnel (notamment météorologique), il peut également référer à un sujet phrastique ou indéterminé, c'est-à-dire qu'il correspond à la fois au $i l$ de fr. il pleut, il faut... et au ça de ça marche, ça m'est égal...

${ }^{15}$ Cette tendance est d'autant plus accentuée dans le registre familier où on + P3 se substitue à nous + P4.
} 
tendance (plus accentuée en piémontais) au syncrétisme entre les pers. 1 et 3, 2 et 5,4 et 6 , comme le montrent les tableaux suivants représentant la structure de la flexion des verbes 'parler' et 'vendre' (les personnes ayant une forme spécifique sont figurées par un tiret, les personnes syncrétiques par une lettre majuscule ; il est tenu compte tant du syncrétisme vertical que du syncrétisme horizontal) :

Tableau 7 : Ramats, structure de la flexion verbale

\begin{tabular}{|c|c|c|c|c|c|c|c|}
\hline & $\begin{array}{c}\text { Ind. } \\
\text { prés. }\end{array}$ & $\begin{array}{l}\text { Imp. } \\
\text { gr.1 }\end{array}$ & $\begin{array}{c}\text { Imp. } \\
\text { gr. 2, 3 }\end{array}$ & Cond. & Futur. & $\begin{array}{c}\text { Subj. } \\
\text { prés. }\end{array}$ & $\begin{array}{c}\text { Subj. } \\
\text { imp. }\end{array}$ \\
\hline P1 & - & - & C ou - & F ou - & I & A & M \\
\hline P2 & A & - & D & G & I & K & N \\
\hline P3 & - & - & C & F & I & A & M \\
\hline P4 & - & B & E & H & J & L & O \\
\hline P5 & - & - & D & G & I & K & N \\
\hline P6 & - & B & E & H & J & L & O \\
\hline
\end{tabular}

Tableau 8 : piémontais, structure de la flexion verbale ${ }^{16}$

\begin{tabular}{|c|c|c|c|c|c|c|c|}
\hline & $\begin{array}{c}\text { Ind. } \\
\text { Prés. }\end{array}$ & $\begin{array}{l}\text { Imp. } \\
\text { gr. }\end{array}$ & $\begin{array}{c}\text { Imp. } \\
\text { gr. 2, 3 }\end{array}$ & Cond. & Futur. & $\begin{array}{c}\text { Subj. } \\
\text { prés. }\end{array}$ & $\begin{array}{c}\text { Subj. } \\
\text { imp. }\end{array}$ \\
\hline P1 & A & D & G & J & - & C & M \\
\hline P2 & B & E & H & K & - & B & N \\
\hline P3 & C & D & G & J & - & C & M \\
\hline P4 & - & F & I & L & - & A & O \\
\hline P5 & B & E & H & K & - & B & N \\
\hline P6 & A & F & I & L & - & A & O \\
\hline
\end{tabular}

Dans le parler des Chiomonte et de Ramats, aucune forme verbale pourvue de son clitique sujet n'est homophone avec une autre forme. En piémontais cela ne se produit que dans un cas : la pers. 1 du présent de l'indicatif et la pers. $4 \mathrm{du}$ présent du subjonctif : [i k'antu] = 'je chante' ou '(que) nous chantions'.

Dans l'occitan de Chiomonte et des Ramats, ainsi qu'en piémontais turinois, en l'absence d'un clitique ou d'une désinence spécifiques, c'est l'association du clitique et de la désinence qui permet d'identifier la personne du verbe, ce qui peut être représenté par les schémas donnés en annexe (annexe 1) qui symbolisent la flexion de l'imparfait de 'vendre' en piémontais turinois et du subjonctif présent de ce même verbe en occitan des Ramats.

\subsection{Omission éventuelle du clitique}

Si l'on analyse le fonctionnement des différents systèmes étudiés d'un point de vue morphosyntaxique, il apparait que les conditions éventuelles permettant ou

16 Source : Grosso 2000. 
exigeant l'omission du clitique, et la fréquence d'omission de celui-ci, sont différentes dans les trois systèmes.

\subsubsection{Piémontais}

En piémontais turinois, le clitique est obligatoire quel que soit le contexte, y compris en présence d'un sujet lexical ou après un relatif sujet : Nosgnor e San Pé a caminavo fin da la matin 'Notre Seigneur et saint Pierre marchaient depuis

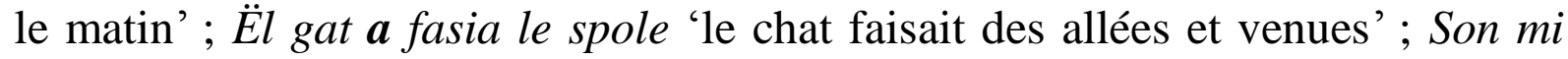
che i't parlo 'C'est moi qui te parle'; J'era'd rat ch'a fasio rubaté tome e tomin e ch'a possavo'd barlèt pien ëd vin 'Il y avait des rats qui faisaient dégringoler tommes et "tommines" [tommes fraiches] et poussaient des barils pleins de vin' (Brero / Bertodatti 2000, 185, 210, 81, 210)

\subsubsection{Français standard}

En français standard le clitique sujet ne double pas le sujet lexical : Pierre est arrivé et non *Pierre il est arrivé. Son omission est possible dans le cas de syntagmes verbaux coordonnés ou juxtaposés : Nous mangeons et buvons ; Il allait, venait, repartait. Enfin il est obligatoirement omis après un relatif sujet : L'homme qui marche et non *L'homme qu'il marche.

\subsubsection{Occitan de Chiomonte et des Ramats}

Dans l'occitan de Chiomonte et des Ramats, le clitique sujet double fréquemment le sujet lexical, mais ce n'est pas obligatoire. De plus on observe des variations considérables de la fréquence d'omission suivant les locuteurs. Nous avons procédé à un comptage sur des données enregistrées provenant de quatre locuteurs: le questionnaire de l'ASIt (Atlante Sintattico d'Italia) enregistré en septembre 2011 auprès d'une locutrice des Ramats (locutrice 1) née en 1961 (environ 4500 mots), et des extraits d'entretiens d'environ 2000 mots chacun (non comprises les interventions de l'intervieweur) réalisés en 1984 auprès de trois locuteurs nés respectivement en 1895 (locutrice 2), 1905 (locutrice 3), 1897 (locuteur 4) ${ }^{17}$. Les résultats obtenus, en termes d'omission du clitique en présence d'un sujet lexical à gauche du verbe, sont les suivants :

- Locutrice 1: 4 occurrences sur 112, soit 3,5\%

- Locutrice 2: 6 occurrences sur 21, soit $29 \%$

- Locutrice $3: 2$ occurrences sur 18, soit $11 \%$

- Locuteur $4: 9$ occurrences sur 9, soit $100 \%$

${ }^{17}$ Les locuteurs 1 et 4 sont originaires des Ramats, les locutrices 2 et 3 du bourg de Chiomonte. 
Les données concernant la locutrice 1 sont des données élicitées sous formes de phrases prononcées en italien par l'enquêteur et traduites en dialecte par l'informatrice, alors que pour les autres locuteurs il s'agit de discours spontané. Or, en situation d'élicitation les locuteurs ont tendance à donner les formes les plus marquées. C'est pourquoi les données émanant de la locutrice 1 ne sont probablement pas représentatives du discours spontané. En outre, le faible nombre d'occurrences chez les autres locuteurs - en particulier chez le locuteur 4 - ne permet pas de généralisation fine, néanmoins ces données sont révélatrices d'une importante variation ${ }^{18}$.

Après un relatif sujet, le clitique peut être omis, mais le phénomène n'est pas massif.

Quel que soit le contexte, la fréquence d'omission du clitique sujet est variable suivant les personnes du verbe : le clitique est souvent omis aux pers. 1 et 4 , parfois aux pers. 5 et 6 , très rarement aux pers. 2 et 3 , mais cela n'est pas totalement impossible. Le seul cas où, dans le corpus, nous n'avons trouvé aucune occurrence d'omission du clitique est celui du clitique de pers. 3 masculin ou féminin (mais pas neutre), en tête d'énoncé.

Le fait qu'une forme verbale soit syncrétique n'empêche pas l'omission du clitique. En d'autres termes, la personne peut être sous-spécifiée. Par exemple, dans le parler des Ramats, on a [a parl'avã] 'nous parlions', [i parl'avã] 'ils parlaient', [la: parl'avã] 'elles parlaient', mais [parl'avã] employé sans clitique vaudra pour 'nous parlions' ou 'ils/elles parlaient'. Dans ce cas le sens peut généralement se déduire du contexte phrastique, discursif ou pragmatique.

\section{Synthèse}

Nous pouvons maintenant procéder à une synthèse des différents phénomènes que nous avons examinés.

En piémontais - et en bonifacien à la pers. 2 - le clitique est strictement obligatoire quel que soit le contexte. Dans le parler de Saorge (aux pers. 2 et 3) et en français, il est obligatoire, sauf contextes particuliers autorisant ou exigeant son omission. Dans le parler de Chiomonte et des Ramats, sa présence est massive - avec d'importantes variations de fréquence suivant les personnes du verbe, le contexte, les locuteurs -, mais il n'est pas strictement obligatoire.

L'omission du clitique sujet en présence d'un sujet lexical est obligatoire en français standard et dans le parler de Saorge, possible dans l'occitan de Chiomonte et des Ramats, avec d'importantes variations de fréquence suivant

\footnotetext{
${ }^{18}$ La transcription et l'analyse d'un volume plus important de données - notamment des données collectées en 2012 auprès d'autres locuteurs - permettront d'affiner l'analyse.
} 
les locuteurs, et impossible en piémontais (la question est sans objet pour le bonifacien).

L'omission du clitique sujet après un relatif sujet est obligatoire en français standard et dans le parler de Saorge, possible dans l'occitan de Chiomonte et des Ramats, sans que le phénomène soit massif, et impossible en piémontais et (pour la pers. 2) en bonifacien.

Avec une forme verbale syncrétique, lorsqu'il existe un clitique sujet, la personne peut être sous-spécifiée dans l'occitan de Chiomonte et des Ramats, ce qui est impossible dans les autres variétés étudiées.

\section{Conclusion}

Dans les systèmes présentant du syncrétisme à la fois dans les désinences verbales et dans le paradigme des clitiques sujets, c'est l'association des marques qui produit le marquage : c'est-à-dire que le marquage d'un trait grammatical (personne, temps, mode) peut mettre en jeu plusieurs marques de natures différentes (clitique, affixale, suprasegmentale, lexicale...). Ce fait ne peut pas être interprété en termes de morphème discontinu, comme par exemple cela est possible pour la morphologie nominale des langues berbères : kabyle $a$-mazi - $\emptyset$ 'berbère' (m.), ta-mazi - $t$ 'berbère' (f.), i-maziy-en 'berbères' (m.), ti-maziy-int 'berbères' (f.). En effet pour qu'on puisse parler de morphèmes discontinus, il faudrait que les deux composants du morphème soient toujours identiques et de même statut, comme c'est le cas pour les morphèmes discontinus du berbère. Or, dans le cas qui nous occupe, on a deux éléments de statuts différents (un clitique et un suffixe) dont le premier varie uniquement en fonction de la personne et le second en fonction de la personne, du temps et du mode.

Entre systèmes strictement pro drop et systèmes strictement non pro drop, il existe un continuum dans lequel plusieurs variables entrent en jeu :

- le nombre de clitiques

- les conditions éventuelles autorisant ou exigeant l'omission du clitique.

- la plus ou moins grande fréquence d'omission lorsque celle-ci est possible (d'un système à l'autre, d'une personne du verbe à l'autre dans un même système, d'un individu à l'autre dans une même communauté linguistique).

Ces observations nous semblent confirmer le fait qu'il n'existe pas de distinction "robuste" entre pro drop et non pro drop, ainsi que le souligne Heap (2000, 152153) :

Tout d'abord, il doit être très clair que l'idée du paramètre du sujet nul n'est plus viable du tout. Il ne s'agit pas là d'une conclusion originale - on annonce sa mort 
depuis une décennie déjà - mais il vaut la peine de se convaincre de ce fait. D'autres études le démontrent mieux que la nôtre ne peut le faire : le paramètre au sens large, comme corrélation de propriétés apparemment indépendantes, ne peut pas tenir debout (Safir 1985 ; Roberge 1989 ; Wanner 1993). Il n'est plus question ici que du paramètre dans le sens le plus étroitement immédiat, comme outil descriptif permettant d'opposer deux classes de grammaires naturelles. Or même à ce niveau descriptif, le paramètre du sujet nul n'a qu'une utilité très limitée : en plus des réglages binaires [ \pm sujet nul], il faudrait au moins une bonne douzaine de réglages intermédiaires correspondant aux paradigmes partiels dans notre typologie [...]. Appeler «paramètre» une dimension grammaticale qui admet toutes ces valeurs scalaires, c'est garder la terminologie d'un modèle sans y attribuer un contenu réel.

Enfin, d'un point de vue méthodologique, nous croyons fondamental, dans les cas où du syncrétisme apparaît à la fois dans les désinences et dans le paradigme des clitiques sujets, d'analyser le système clitiques-désinences comme un tout constituant un seul et même système de marquage.

\section{Bibliographie}

Adams, Marianne (1988): Les effets V2 en ancien et en moyen français. Dans : Revue québécoise de linguistique théorique et appliquée 7/3, 13-39

Saint-Denis : Presses Universitaires de Vincennes

Battye, Alan / Roberts Ian (éds) (1995) : Clause Structure and Language Change, Oxford: Oxford University Press.

Botton, Charles / Gaber, Jean / Bianco, Albert (2000) : Eu saoueudjinn. Le parler de Saorge. Breil-sur-Roya : Éditions du Cabri

Brero, Camillo / Bertodatti, Remo (2000) : Grammatica della lingua piemontese. Parola vita-letteratura. Turin : Editrice artistica piemontese

Cabredo-Hofherr, Patricia (2004) : Les clitiques sujets du français et le paramètre du sujet nul. Dans : Langue française 141, 99-109

Comiti, Jean-Marie (1994) : Bunifazziu e a se lengua - Bonifacio et sa langue. Ajaccio : Ed. Squadra di u Finusellu

D’Alessandro, Roberta / Ledgeway, Adam / Roberts, Ian (éds.) (2019) : Syntactic variation. The dialects of Italy. Cambridge : Cambridge University Press

De Cat, Cécile (2005) : French subject clitics are not agreement markers. Dans: Lingua 115, 1195-1219

Dalbera, Jean-Philippe (1991): Les pronoms personnels atones dans les parlers des AlpesMaritimes. Champs et mécanismes de variation. Dans : Kremer, 599-613

Dalbera Jean-Philippe (1994) : Les parlers des Alpes-Maritimes : étude comparative, essai de reconstruction. Londres : Association Internationale d'Etudes Occitanes - University of London

Floricic Franck / Molinu Lucia (2008) : L'Italie et ses dialectes. Dans : Lalies 28, 7-107

Godard, Danièle (éd.) (2003) : Les langues romanes : Problèmes de la phrase simple. Paris : CNRS éditions 
Grosso, Michela (2000) : Grammatica essenziale della lingua piemontese. Turin : Libreria piemontese

Heap, David (2000) : La variation grammaticale en géolinguistique : les pronoms sujet en roman central. Munich : LINCOM Europa

Jaeggli Osvaldo / Silva-Corvalán, Carment (éds.) (1986) : Studies in Romance. Dordrecht : Foris

Kayne, Richard (1983): Chaînes, catégories extérieures à $S$ et inversion complexe en français. Dans : Langue française 58, 36-65

Kremer, Dieter (éd.) (1991) : Actes du XVIII Congrès International de Linguistique et de Philologie Romanes. Tübingen : Niemeyer

Manzini, Rita / Savoia, Leonardo (2005): I dialetti italiani e romanci. Morfosintassi generativa. Alessandria : Edizioni dell'Orso.

Manzini, Rita / Savoia, Leonardo (2010) : Syncretism and suppletivism in clitic systems : underspecification, silent clitics or neither ? Dans : D'Alessandro et al., 86-101

Meyer-Lübke, Wilhelm (1895) : Grammaire des langues romanes (tome II). Paris : Welter

Miller, Philip / Monachesi, Paola (2003) : Les pronoms clitiques dans les langues romanes. Dans : Godard, 67-123

Poletto, Cecilia (1993) : La sintassi de soggetto nei dialetti italiani settentrionali. Padova : Unipress.

Poletto, Cecilia (1995) : The diachronic development of subject clitics in north eastern Italian dialects, Clause structure and language change, dans : A.Battye and I. Roberts (éds), 295-324.

Raible, Wolfgang / Oesterreicher Wulf (éds.) (1993) : Actes du XX Congrés International de Linguistique et de Philologie Romanes, Tome III-lV (Typologie des langues romanes). Bruxelles : De Gruyter

Rizzi, Luigi (1986) : On the status of subject clitics in Romance. Dans : Jaeggli / SilvaCorvalán, 391-419

Roberge, Yves (1989): Les paramètres de la variation. Dans : Journal of the Atlantic Provinces Linguistics Association 11, 94-114

Safir, Ken (1985) : Syntactic Chaim. Cambridge : Cambridge University Press

Savoia, Leonardo / Manzini, Rita (2010) : Les clitiques sujets dans les variétés occitanes et francoprovençales italiennes. Dans : Corpus 9, 165-189

Vanelli, Laura (1998): I dialetti italiani settentrionali nel panorama romanzo. Studi di sintassi e morfologia, Roma : Bulzoni.

Wanner, Dieter (1993) : L'expression du sujet dans les langues romanes. Dans : Raible / Oesterreicher, 449-460

Zribi-Hertz, Anne (1994) : La syntaxe des clitiques nominatifs en français standard et avancé. Dans : Travaux de linguistique et de philologie 32, 131-147

Jean SIBILLE,

Laboratoire CLLE-ERSS (UMR 5263)

CNRS / Université de Toulouse II - Le Mirail

5 allées Antonio Machado,

31058 Toulouse cedex 9 (France)

jsibille@univ-tlse2.fr 
Annexe 1

Figure 1 : piémontais, imparfait de [v'ende] 'vendre'

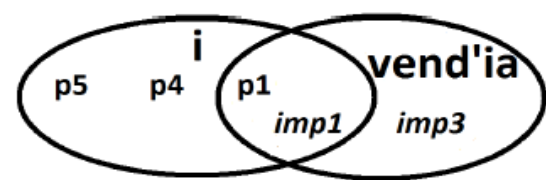

'je vendais'

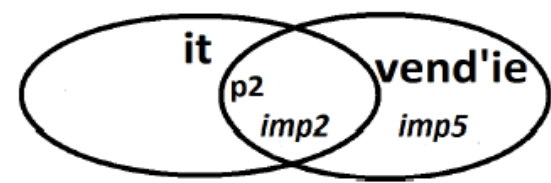

'tu vendais'

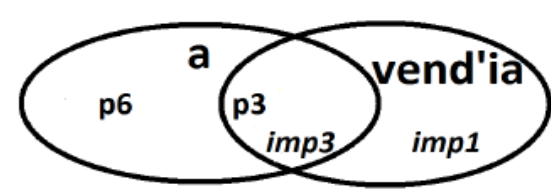

'il/elle vendait'

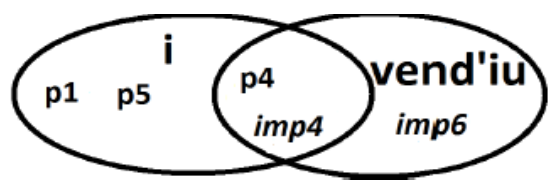

'nous vendions'
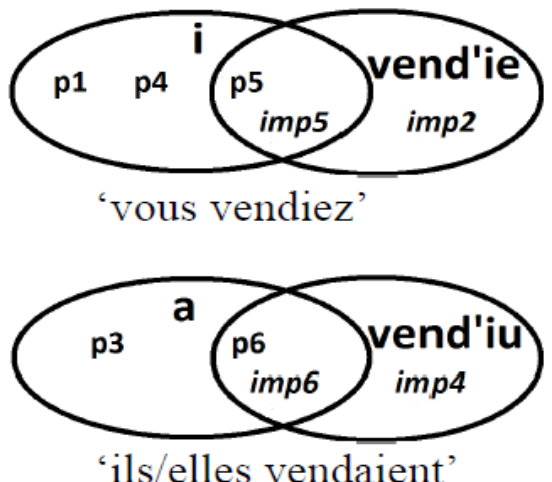

Figure 2 : Ramats, subjonctif présent de [v'ande] 'vendre'

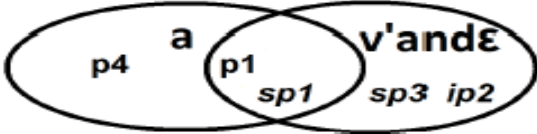

'(que) je vende'

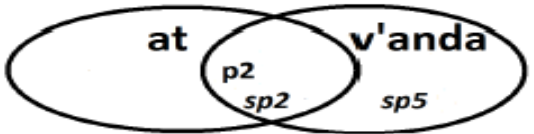

'(que) tu vendes'

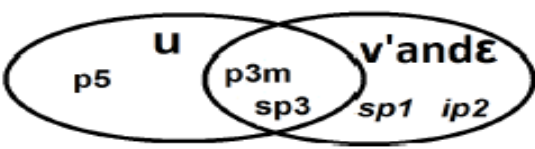

'(qu')il vende'

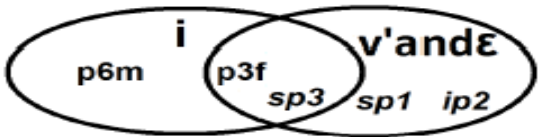

'(qu')elle vende'

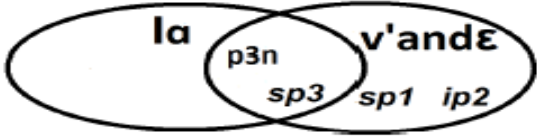

'(que) ça vende'
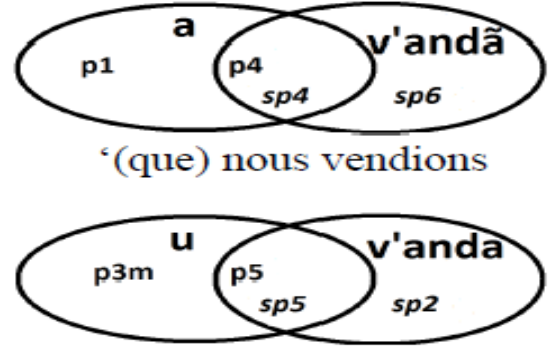

'(que) vous vendiez'
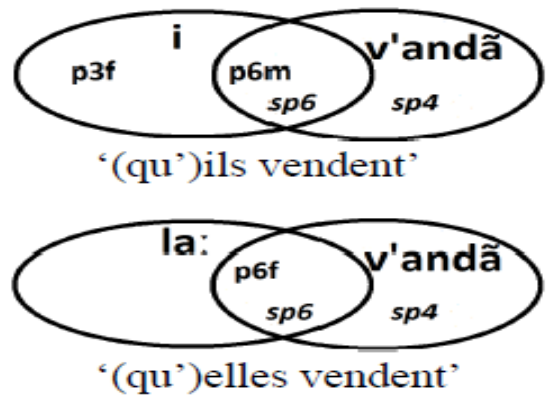

Légende : $\quad \mathrm{p} 1=$ personne $1 ; \mathrm{p} 2=$ personne 2 etc.

impl $=$ imparfait pers. 1 ; imp $2=$ imparfait pers. 2 ; etc.

$s p 1=$ subjonctif présent personne 1 ; etc. ip $2=$ indicatif présent pers. 2 